\title{
Intrinsic androgen-dependent gene expression patterns revealed by comparison of genital fibroblasts from normal males and individuals with complete and partial androgen insensitivity syndrome Paul-Martin Holterhus*1, Uta Deppe ${ }^{2}$, Ralf Werner ${ }^{2}$, Annette Richter-Unruh ${ }^{3}$, Jan-Hendrik Bebermeier ${ }^{2}$, Lutz Wünsch ${ }^{4}$, Susanne Krege ${ }^{5}$, Hans- Udo Schweikert ${ }^{6}$, Janos Demeter ${ }^{7}$, Felix Riepe ${ }^{1}$, Olaf Hiort ${ }^{2}$ and James D Brooks ${ }^{8}$
}

Address: ${ }^{1}$ Department of Pediatrics, University-Hospital Schleswig-Holstein, Campus Kiel, Schwanenweg 20, Kiel, Germany, ${ }^{2}$ Department of Pediatric and Adolescent Medicine, University-Hospital Schleswig-Holstein, Campus Lübeck, Ratzeburger Allee 160, Lübeck, Germany, ${ }^{3}$ Endokrinologikum Ruhr, Alter Markt 4, Bochum, Germany, ${ }^{4}$ Department of Pediatric Surgery, University Hospital Schleswig-Holstein, Campus Lübeck, Ratzeburger Allee 160, Lübeck, Germany, ${ }^{5}$ Department of Urology, University of Essen, Essen, Germany, ${ }^{6}$ Department of Internal Medicine, University of Bonn, Bonn, Germany, ${ }^{7}$ Department of Genetics, Stanford University School of Medicine, CA, USA and ${ }^{8}$ Department of Urology, Stanford University School of Medicine, CA, USA

Email: Paul-Martin Holterhus* - holterhus@pediatrics.uni-kiel.de; Uta Deppe - Uta_Deppe@web.de; Ralf Werner -werner@paedia.ukl.muluebeck.de; Annette Richter-Unruh - annette.richterunruh@endokrinologikum.com; Jan-Hendrik Bebermeier - bebermeier@paedia.ukl.muluebeck.de; Lutz Wünsch - wuensch@medinf.mu-luebeck.de; Susanne Krege - susanne.krege@uni-essen.de; Hans-

Udo Schweikert - umm506@uni-bonn.de; Janos Demeter - jdemeter@genome.stanford.edu; Felix Riepe - friepe@pediatrics.uni-kiel.de; Olaf Hiort - hiort@paedia.ukl.mu-luebeck.de; James D Brooks - jdbrooks@stanford.edu

* Corresponding author

Published: 18 October 2007

BMC Genomics 2007, 8:376 doi:10.1/86/147|-2164-8-376
Received: 16 February 2007

Accepted: 18 October 2007

This article is available from: http://www.biomedcentral.com/I47I-2/64/8/376

(C) 2007 Holterhus et al; licensee BioMed Central Ltd.

This is an Open Access article distributed under the terms of the Creative Commons Attribution License (http://creativecommons.org/licenses/by/2.0), which permits unrestricted use, distribution, and reproduction in any medium, provided the original work is properly cited.

\begin{abstract}
Background: To better understand the molecular programs of normal and abnormal genital development, clear-cut definition of androgen-dependent gene expression patterns, without the influence of genotype (46, XX vs. 46, $X Y$ ), is warranted. Previously, we have identified global gene expression profiles in genital-derived fibroblasts that differ between $46, X Y$ males and 46, XY females with complete androgen insensitivity syndrome (CAIS) due to inactivating mutations of the androgen receptor (AR). While these differences could be due to cell autonomous changes in gene expression induced by androgen programming, recent work suggests they could also be influenced by the location from which the fibroblasts were harvested (topology). To minimize the influence of topology, we compared gene expression patterns of fibroblasts derived from identical urogenital anlagen: the scrotum in normally virilized $46, X Y$ males and the labia majora from completely feminized $46, X Y$ individuals with CAIS.

Results: 612 transcripts representing 440 unique genes differed significantly in expression levels between scrotum and CAIS labia majora, suggesting the effects of androgen programming. While some genes coincided with those we had identified previously (TBX3, IGFBP5, EGFR, CSPG2), a significant number did not, implying that topology had influenced gene expression in our previous experiments. Supervised clustering of gene expression data derived from a large set of fibroblast cultures from individuals with partial AIS revealed that the new, topology controlled data set better classified the specimens.

Conclusion: Inactivating mutations of the AR, in themselves, appear to induce lasting changes in gene expression in cultured fibroblasts, independent of topology and genotype. Genes identified are likely to be relevant candidates to decipher androgendependent normal and abnormal genital development.
\end{abstract}




\section{Background}

Androgen receptor (AR) signaling is the key determinant of virilization in male external genitalia development [14]. Its importance is highlighted in the androgen insensitivity syndrome (AIS), a virtual human AR "knock-out" due to inactivating mutations of the AR gene, and characterized by defects in virilization of $46, \mathrm{XY}$ individuals despite normal or elevated serum testosterone levels. The phenotype can range from normal female in complete AIS (CAIS), to lesser degrees of genital ambiguity in partial AIS (PAIS) in which the degree of virilization is related to the degree of AR function [2,3]. Genital masculinization involves a comprehensive re-organization of genital anatomy in which androgens induce a permanent male developmental fate in the originally bipotent anlagen. For example, in response to embryonal androgen signaling, the labioscrotal swellings will develop into a scrotum and not into labia majora (as overviewed by [5]). These observations indicate the existence of androgen-mediated gene expression programs that are responsible for implementation and persistence of male-specific genital morphology and function. In general, distinguishing X- and Y-chromosomal influences from hormonal influences on genital development has proven difficult $[6,7]$; however, CAIS, in which all individuals possess an $\mathrm{XY}$ genotype, represents the ideal situation for focusing on the role of androgens.

In a previous study, we reported evidence for androgenmediated programming of gene expression by comparing genital skin fibroblasts from normal males to those derived from CAIS individuals [8]. However, the demonstration that cultured fibroblasts show stable transcription signatures that reflect their site of origin in mature adult tissues (transcriptional topographic memory) [9] raises the possibility that fibroblast transcript signatures might also reflect androgen-independent aspects of their developmental history. Were that the case, the transcript signatures we had identified might be influenced both by androgen signaling during genital development and topology. Even though the normal male and CAIS fibroblasts were both derived from the genital skin, their precise sites of biopsy differed both topologically and with regard to embryological origin. The normal male genital fibroblasts were grown from biopsies of the foreskin (derived from the genital tubercle), while CAIS fibroblasts came from biopsies of the labia majora (derived from the labioscrotal swellings) [8]. To better define the gene expression programs dependent only on developmental androgen actions, we analyzed gene expression profiles of fibroblasts derived from homologous embryonic structures: the labioscrotal swellings in $46, \mathrm{XY}$ males (scrotum) and 46, XY females (labia majora). We validated the biological relevance of this approach by comparing the ability of this new gene expression set and the previous set to classify a large set of individuals representing the whole clinical spectrum of AIS phenotypes based on gene expression profiles alone.

\section{Results \\ Identification of a topology-independent, AR-dependent gene expression program}

We had previously identified an AR-dependent gene expression signature by comparing normal male foreskin fibroblasts to those cultured from diverse sites in the genitals of patients with documented CAIS. Subsequent reports that cultured fibroblasts retain topographic transcriptional memory (gene expression signatures that reflect their site of biopsy) led to concerns that the ARdependent gene expression signature we identified could have been affected by topological differences in the fibroblast samples used. To test for this potential confounder, we repeated microarray experiments on seven independent strains of normal male scrotal fibroblasts (S1, S4, S5, S8, S9, S11, and S12) and duplicate samples of four labia majora fibroblasts derived from $46, \mathrm{XY}$ individuals with CAIS due to proven inactivating mutations of the AR-gene. Both fibroblasts were derived from identical anlagen: the labioscrotal swellings. The SAM procedure revealed 612 transcripts representing 440 unique genes that differed significantly in expression level between the groups at a false discovery rate of 0.038 (Figure 1).

The new topology-controlled data set showed some similarities to the AR-dependent gene set we had identified previously, with 42 unique transcripts found in both gene sets, including 34 transcripts that were up-regulated in normal male derived fibroblasts, and 8 up-regulated in the CAIS female-derived fibroblasts. Genes up-regulated in normal male fibroblasts in both data sets included TBX3 (T-box 3), CBX6 (Chromobox homologue 6), IGFBP5 (Insulin-like growth factor binding protein 5), and EGFR (epidermal growth factor receptor) while several others were no longer identified as significantly different between the data sets, such as TBX2 (T-box 2), TBX5 (T-box 5), BMP4 (bone morphogenetic protein 4), HOXA13 (Homeobox A13), WNT2 (Wingless-type MMTV integration site family, member 2), and FOXF2 (Forkheadbox F2). The significant change in the gene lists strongly suggested that topology influenced the gene expression signatures identified in our original series of experiments.

\section{Topology independent AR gene expression program classifies diverse AIS samples}

To evaluate the relevance of the topology-controlled, AR dependent gene list, we tested its ability to classify 72 microarray experiments performed on fibroblast samples derived from 51 individuals that included normal males, normal females, and individuals with PAIS and CAIS (Fig. 2 and table 1). AIS samples were graded according to the 


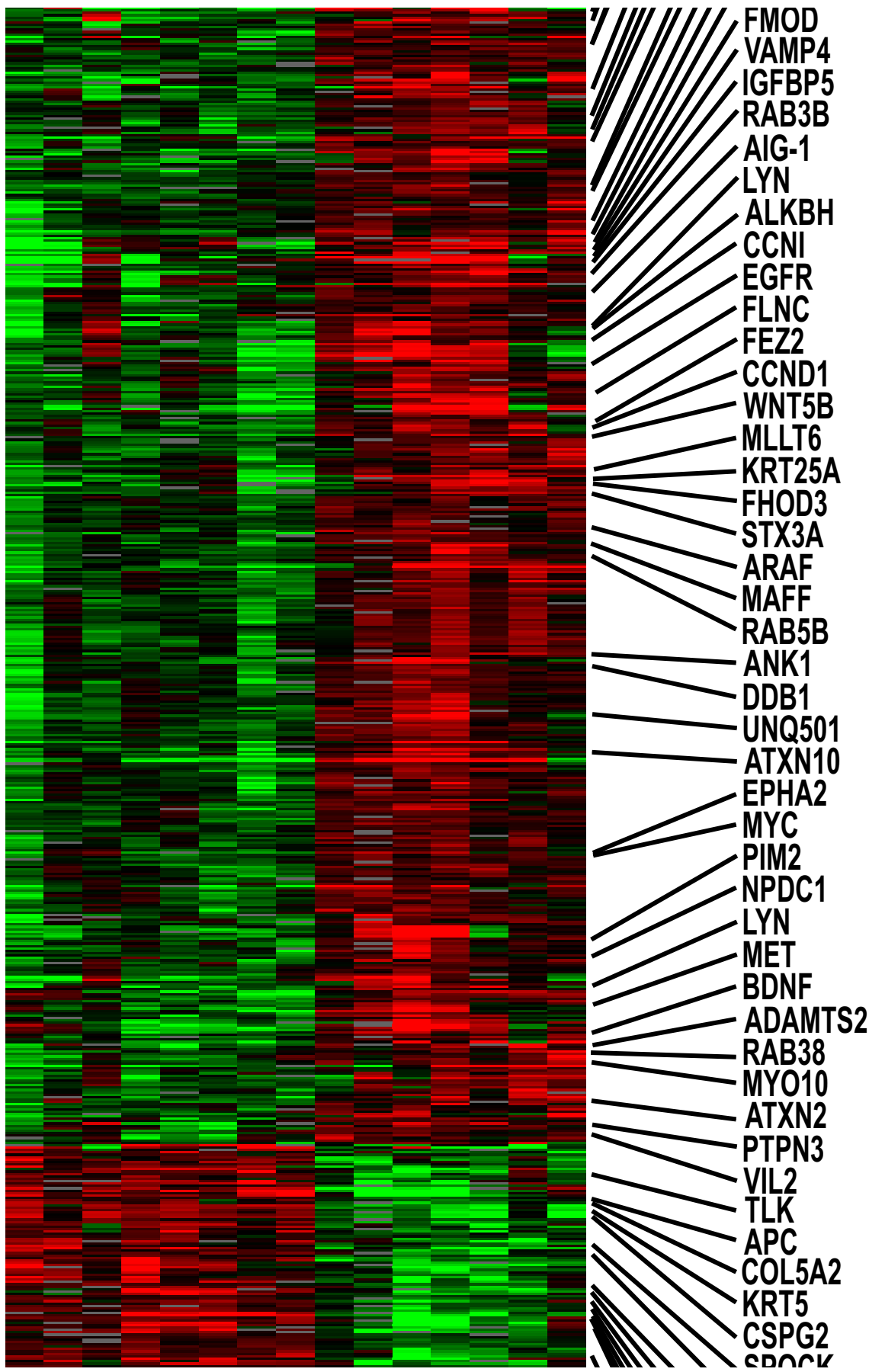

Figure I

Transcripts with significant differences of expression levels between normal scrotum and CAIS labia majora. Transcript levels of 612 genes identified by SAM analysis as differing between fibroblasts derived from normal male scrotum (green) and labia majora of 46, XY females with CAIS (pink). Individual transcripts are grouped by hierarchical cluster analysis and are displayed in rows while experiments are represented in columns. Expression values per gene are centered by the mean $\log _{2}$ red/green normalized ratio. Increasing red intensity corresponds to higher relative transcript levels compared to the mean expression level across all 15 array experiments. Increasing green intensity corresponds to relatively decreased transcript levels compared to the mean. On the right side, examples of individual genes (gene symbols according to S.O.U.R.C.E. [24]) discussed in the paper or falling into the biological processes and cellular pathways detected by PANTHER are displayed. Detailed data on figure $I$ is available in additional files I, 2, 3, 4 . 

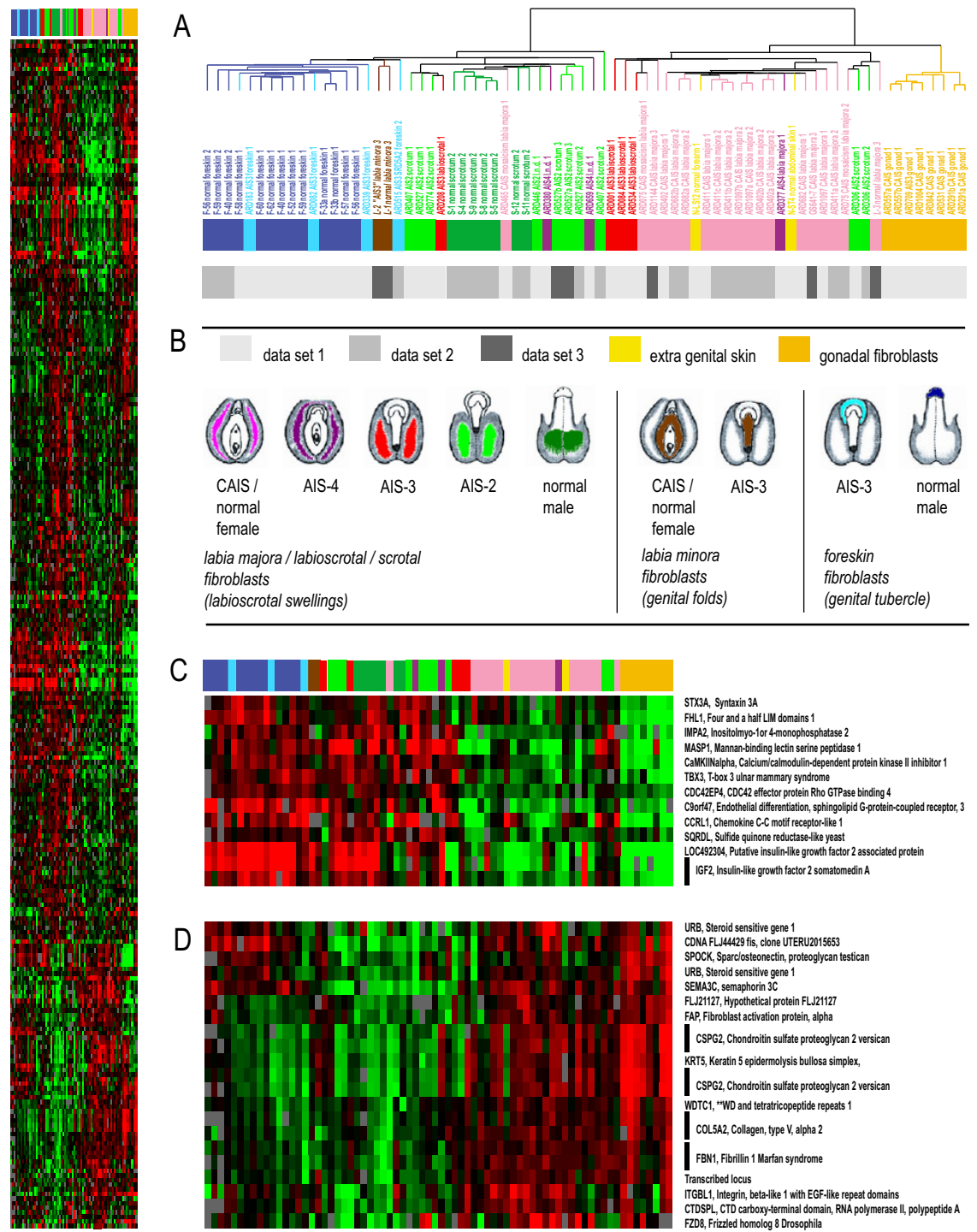

Figure 2

Cluster analysis of normal male fibroblasts from scrotum and foreskin as well as 46, XY individuals with PAIS and CAIS. Hierarchical clustering analysis of 72 microarray experiments of cultured genital fibroblasts using the SAM derived gene list. The heatmap on the left displays 259 genes that had at least $85 \%$ interpretable data across the experiments whose expression levels were at least 2-fold different from the mean expression across all samples in at least 5 microarrays. (A) The cluster dendrogram demonstrating the degree of relatedness (Pearson correlation) between the expression patterns of the 259 genes in the cultured fibroblast samples. The length of the arms of the dendrogram reflects the degree of correlation between the samples. Samples are color coded to reflect the localization of the biopsy and the degree of external genital virilization according to a grading scheme developed by Sinnecker et al. [10]. The grey bar below indicates whether a sample was derived from dataset I, 2 or 3. "L.n.d." signifies that the biopsy localization was not accurately documented. Italics indicate a sample with a 46, XX karyotype. (B) Schematic depiction of the external genitalia phenotype of the cases from which the fibroblast cultures were derived using color coding that corresponds to the degree of genital ambiguity and the location of the biopsy. Color coding corresponds to the bar below the dendrogram in (A). (C) Cluster of AR-dependent transcripts that are highly expressed in the left "male" major branch of the cluster that are expressed at significantly lower levels in the "female" branch of the cluster on the right. TBX3, previously reported in ulnar mammary syndrome and IGF2, previously reported as being down-regulated in CAIS [20] are shown in this cluster. (D) Cluster of AR-dependent transcripts that are expressed at significantly lower levels in the lefthand "male" branch of the cluster and at higher levels in the righthand "female" branch. These include many extracellular matrix genes such as proteoglycan testican, versican, and fibrillin I. Detailed data on figure 2 is available in additional files $5,6,7,8$. 
Table I: Fibroblast strains

\begin{tabular}{|c|c|c|c|c|c|}
\hline cell strain & $\begin{array}{l}\text { micro-array } \\
\text { data set }\end{array}$ & $\begin{array}{l}\text { anatomic origin } \\
\text { embryonal anlagen }\end{array}$ & $\begin{array}{l}\text { external } \\
\text { genitalia AIS-stage }\end{array}$ & $\begin{array}{l}\text { age at biopsy } \\
\text { years; months }\end{array}$ & $\begin{array}{l}\text { selected functional data (androgen } \\
\text { binding, AR-genotype, specific } \\
\text { remarks) }\end{array}$ \\
\hline F-33 & I & foreskin genital tubercle & normal fertile male & $51 ; 11$ & $\mathrm{~K}_{\mathrm{d}} 0.08 \mathrm{nM}, \mathrm{B}_{\max } 26 \mathrm{fmol} / \mathrm{mg}$ protein \\
\hline F-40 & 1,2 & foreskin genital tubercle & normal male & $7 ; 11$ & $\mathrm{~K}_{\mathrm{d}} 0.09 \mathrm{nM}, \mathrm{B}_{\max } 32 \mathrm{fmol} / \mathrm{mg}$ protein \\
\hline F-52 & 1 & foreskin genital tubercle & normal male & $0 ; 3$ & $\mathrm{~K}_{\mathrm{d}} 0.06 \mathrm{nM}, \mathrm{B}_{\max } 35 \mathrm{fmol} / \mathrm{mg}$ protein \\
\hline F-56 & I, 2 & foreskin genital tubercle & normal fertile male & $42 ; 10$ & $\mathrm{~K}_{\mathrm{d}} 0.07 \mathrm{nM}, \mathrm{B}_{\max } 13 \mathrm{fmol} / \mathrm{mg}$ protein \\
\hline F-57 & 1 & foreskin genital tubercle & normal male & $5 ; 11$ & $\mathrm{~K}_{\mathrm{d}} 0.05 \mathrm{nM}, \mathrm{B}_{\max } 24 \mathrm{fmol} / \mathrm{mg}$ protein \\
\hline F-58 & I & foreskin genital tubercle & normal male & $5 ; 11$ & $\mathrm{~K}_{\mathrm{d}} 0.10 \mathrm{nM}, \mathrm{B}_{\max } 31 \mathrm{fmol} / \mathrm{mg}$ protein \\
\hline F-59 & 1,2 & foreskin genital tubercle & normal male & $0 ; 8$ & $\mathrm{~K}_{\mathrm{d}} 0.07 \mathrm{nM}, \mathrm{B}_{\max } 40 \mathrm{fmol} / \mathrm{mg}$ protein \\
\hline F-60 & 1 & foreskin genital tubercle & normal male & $5 ; 4$ & $\mathrm{~K}_{\mathrm{d}} 0.05 \mathrm{nM}, \mathrm{B}_{\max } 20 \mathrm{fmol} / \mathrm{mg}$ protein \\
\hline F-62 & I & foreskin genital tubercle & normal male & $2 ; 0$ & $\mathrm{~K}_{\mathrm{d}} 0.08 \mathrm{nM}, \mathrm{B}_{\max } 86 \mathrm{fmol} / \mathrm{mg}$ protein \\
\hline ARD339 & 1 & foreskin genital tubercle & ambiguous (AIS3) & $1 ; 4$ & Glu798Gln \\
\hline ARD082 & I & foreskin genital tubercle & ambiguous (AIS3) & $10 ; 11$ & Asp604Tyr \\
\hline ARD 183 & i & foreskin genital tubercle & ambiguous (AIS3) & $5 ; 1$ & Asp756Ser \\
\hline ARD5I5 & 2 & foreskin genital tubercle & ambiguous (AIS3) & $0 ; 6$ & $\begin{array}{l}\text { homozygous missense mutation Arg227Gln } \\
\text { in SRD5A2 gene leading to } 5 \alpha \text { reductase } \\
\text { type II deficiency }\end{array}$ \\
\hline S-I & 2 & scrotum labioscrotal swellings & normal male & $30 ; 0$ & no functional data \\
\hline S-4 & 2 & scrotum labioscrotal swellings & normal male & $1 ; 3$ & $\mathrm{~K}_{\mathrm{d}} 0.12 \mathrm{nM}, \mathrm{B}_{\max } 44 \mathrm{fmol} / \mathrm{mg}$ protein \\
\hline S-5 & 2 & scrotum labioscrotal swellings & normal male & $\mathrm{I} ; 7$ & $\mathrm{~K}_{\mathrm{d}} 0.09 \mathrm{nM}, \mathrm{B}_{\max } 28 \mathrm{fmol} / \mathrm{mg}$ protein \\
\hline S-8 & 2 & scrotum labioscrotal swellings & normal male & $31 ; 0$ & no functional data \\
\hline S-9 & 2 & scrotum labioscrotal swellings & normal male & $34 ; 0$ & $\mathrm{~K}_{\mathrm{d}} 0.08 \mathrm{nM}, \mathrm{B}_{\max } 55 \mathrm{fmol} / \mathrm{mg}$ protein \\
\hline S-II & 2 & scrotum labioscrotal swellings & normal male & $32 ; 2$ & $\mathrm{~K}_{\mathrm{d}} 0.08 \mathrm{nM}, \mathrm{B}_{\max } 26 \mathrm{fmol} / \mathrm{mg}$ protein \\
\hline S-12 & 2 & scrotum labioscrotal swellings & normal male & $9 ; 2$ & $\mathrm{~K}_{\mathrm{d}} 0.07 \mathrm{nM}, \mathrm{B}_{\max } 37.09 \mathrm{fmol} / \mathrm{mg}$ protein \\
\hline ARD527 & I, 2, 3 & scrotum labioscrotal swellings & $\begin{array}{l}\text { predominantly male } \\
\text { (AIS2) }\end{array}$ & $1 ; 6$ & $\begin{array}{l}\text { Leu7/2Phe, } K_{d} 0.21 \mathrm{nM} ; B_{\max } 38 \mathrm{fmol} / \mathrm{mg} \\
\text { protein }\end{array}$ \\
\hline ARD446 & 1 & scrotum labioscrotal swellings & $\begin{array}{l}\text { predominantly male } \\
\text { (AIS2) }\end{array}$ & $7 ; 8$ & $\begin{array}{l}\text { silent mutation GGG>GGA in codon } 795 \\
\text { of the } A \text { R-gene } K_{d} 0.07 \mathrm{nM}, B_{\max } 26 \mathrm{fmol} / \mathrm{mg} \\
\text { protein }\end{array}$ \\
\hline ARD407 & I, 2 & scrotum labioscrotal swellings & $\begin{array}{l}\text { predominantly male } \\
\text { (AIS2) }\end{array}$ & $1: 0$ & $\begin{array}{l}\text { polyglycin repeat of the } N \text {-terminus } \\
\text { reduced to } 10 \text { repeats plus Ala } 645 \mathrm{Asp}, \mathrm{K}_{\mathrm{d}} \\
0.09 \mathrm{nM} ; \mathrm{B}_{\max } 36 \mathrm{fmol} / \mathrm{mg} \text { protein }\end{array}$ \\
\hline ARD306 & I, 2 & scrotum labioscrotal swellings & $\begin{array}{l}\text { predominantly male } \\
\text { (AIS2) }\end{array}$ & $7 ; 8$ & $\begin{array}{l}\text { Arg855His; } \mathrm{K}_{d} 0.97 \mathrm{nM}, \mathrm{B}_{\max } 20 \mathrm{fmol} / \mathrm{mg} \\
\text { protein }\end{array}$ \\
\hline ARD774 & 1 & scrotum labioscrotal swellings & $\begin{array}{l}\text { predominantly male } \\
\text { (AIS2) }\end{array}$ & $0 ; 11$ & $\begin{array}{l}\text { Ala596Thr; } \mathrm{K}_{\mathrm{d}} 0.08 \mathrm{nM} ; \mathrm{B}_{\max } 43 \mathrm{fmol} / \mathrm{mg} \\
\text { protein }\end{array}$ \\
\hline ARD208 & 1 & $\begin{array}{l}\text { labia majora/scrotum } \\
\text { labioscrotal swellings }\end{array}$ & ambiguous (AIS3) & $\mathrm{I} ; \mathrm{I}$ & $\begin{array}{l}\text { Val746Met; } \mathrm{K}_{\mathrm{d}} 0.16 \mathrm{nM} ; \mathrm{B}_{\max } 3 \mathrm{fmol} / \mathrm{mg} \\
\text { protein }\end{array}$ \\
\hline ARD534 & 1 & $\begin{array}{l}\text { labia majora/scrotum } \\
\text { labioscrotal swellings }\end{array}$ & ambiguous (AIS3) & $2 ; 4$ & Arg608Lys \\
\hline ARD084 & 1 & $\begin{array}{l}\text { labia majora/scrotum } \\
\text { labioscrotal swellings }\end{array}$ & ambiguous (AIS3) & $0 ; 8$ & $\begin{array}{l}\text { no mutation in the whole coding region of } \\
\text { the AR gene, reduced AR-mRNA, reduced } \\
\text { AR-protein and reduced ligand binding ( } \mathrm{K}_{\mathrm{d}} \\
0.05 \mathrm{nM} ; \mathrm{B}_{\max } 6 \mathrm{fmol} / \mathrm{mg} \text { protein) }\end{array}$ \\
\hline ARD00I & I & $\begin{array}{l}\text { labia majora/scrotum } \\
\text { labioscrotal swellings }\end{array}$ & ambiguous (AIS3) & $0 ; 9$ & $\begin{array}{l}\text { exonic splice site mutation AGC>AGT in } \\
\text { codon } 888 \text { leading to aberrant splicing }\end{array}$ \\
\hline ARD380 & 1 & $\begin{array}{l}\text { labia majora/scrotum } \\
\text { labioscrotal swellings }\end{array}$ & ambiguous (AIS3) & $13 ; 1$ & $\begin{array}{l}\text { Arg615Pro, post zygotic mutation leading } \\
\text { to somatic mosaicism }\end{array}$ \\
\hline ARD377 & I & $\begin{array}{l}\text { labia majora/scrotum } \\
\text { labioscrotal swellings }\end{array}$ & $\begin{array}{l}\text { predominantly } \\
\text { female (AIS4) }\end{array}$ & $\mathrm{I} ; 2$ & $\begin{array}{l}\text { lle84ISer, }\left(K_{d} 0.55 \mathrm{nM} ; B_{\max } 17 \mathrm{fmol} / \mathrm{mg}\right. \\
\text { protein), }\end{array}$ \\
\hline ARD659 & I & $\begin{array}{l}\text { labia majora labioscrotal } \\
\text { swellings }\end{array}$ & $\begin{array}{l}\text { Predominantly } \\
\text { female (AIS4) }\end{array}$ & $3 ; 10$ & Ala870Gly \\
\hline ARD 1097 & I, 2 & $\begin{array}{l}\text { labia majora labioscrotal } \\
\text { swellings }\end{array}$ & $\begin{array}{l}\text { normal female } \\
\text { (CAIS) }\end{array}$ & $1 ; 3$ & $\begin{array}{l}\text { Pro390Ser + Arg855Gly, negative androgen } \\
\text { binding }\end{array}$ \\
\hline ARD4I I & I, 2 & $\begin{array}{l}\text { labia majora labioscrotal } \\
\text { swellings }\end{array}$ & $\begin{array}{l}\text { normal female } \\
\text { (CAIS) }\end{array}$ & $0 ; 4$ & Arg855Cys, negative androgen binding \\
\hline ARDII44 & 3 & $\begin{array}{l}\text { labia majora labioscrotal } \\
\text { swellings }\end{array}$ & $\begin{array}{l}\text { normal female } \\
\text { (CAIS) }\end{array}$ & $4 ; 3$ & $\begin{array}{l}\text { pathological androgen binding, } \mathrm{K}_{\mathrm{d}} 1.59 \mathrm{nM} \text {, } \\
\mathrm{B}_{\max } 14 \mathrm{fmol} / \mathrm{mg} \text { protein, no mutation } \\
\text { detected }\end{array}$ \\
\hline $\begin{array}{l}\text { GS64I-7I } \\
\text { ARD29I }\end{array}$ & 3 & $\begin{array}{l}\text { labia majora labioscrotal } \\
\text { swellings }\end{array}$ & $\begin{array}{l}\text { normal female } \\
\text { (CAIS) }\end{array}$ & $18 ; 4$ & Phe794Ser, negative androgen binding \\
\hline
\end{tabular}


Table I: Fibroblast strains (Continued)

\begin{tabular}{|c|c|c|c|c|c|}
\hline ARD682 & 1,2 & $\begin{array}{l}\text { labia majora labioscrotal } \\
\text { swellings }\end{array}$ & $\begin{array}{l}\text { normal female } \\
\text { (CAIS) }\end{array}$ & $14 ; 10$ & $\begin{array}{l}\text { Gln59stop, negative androgen binding, no } \\
\text { AR-protein in Western immunoblot }\end{array}$ \\
\hline ARD402 & 1,2 & $\begin{array}{l}\text { labia majora labioscrotal } \\
\text { swellings }\end{array}$ & $\begin{array}{l}\text { normal female } \\
\text { (CAIS) }\end{array}$ & $\mathrm{I} ; 0$ & $\begin{array}{l}2 \text { base pair deletion in exon I, frameshift, } \\
\text { premature stop codon, negative androgen } \\
\text { binding, very low AR-mRNA transcription, } \\
\text { no AR-protein in Western immunoblot }\end{array}$ \\
\hline ARD075 & 1,2 & $\begin{array}{l}\text { labia majora labioscrotal } \\
\text { swellings }\end{array}$ & $\begin{array}{l}\text { normal female } \\
\text { (CAIS) }\end{array}$ & $12 ; 3$ & $\begin{array}{l}\text { Arg774Cys, post zygotic mutation leading } \\
\text { to somatic mosaicism, } K_{d 1} 0.03 \mathrm{nM} ; B_{\operatorname{maxl}} 2 \\
\text { fmol/mg protein (wilde type AR); } K_{d 2} 8.5 \\
n M ; B_{\max 2} 29 \mathrm{fmol} / \mathrm{mg} \text { protein (mutant AR) }\end{array}$ \\
\hline ARD465 & 1 & $\begin{array}{l}\text { labia majora labioscrotal } \\
\text { swellings }\end{array}$ & $\begin{array}{l}\text { normal female } \\
\text { (CAIS) }\end{array}$ & $5 ; 5$ & $\begin{array}{l}\text { Glu287stop, low expression of wild-type } \\
A R\left(K_{d} 0.1 I \mathrm{nM} ; B_{\max } 4 \mathrm{fmol} / \mathrm{mg} \text { protein), }\right. \\
\text { post-zygotic mutation (somatic mosaicism } \\
\text { likely) }\end{array}$ \\
\hline L-I & 3 & labia minoraurethral folds & normal female & $37 ; 10$ & normal female $(46, X X)$ \\
\hline L-2 & 3 & labia minoraurethral folds & normal female & $23 ; 0$ & $\begin{array}{l}\text { female }(46 X X) \text {, classical congenital adrenal } \\
\text { hyperplasia due to } 2 I \text {-hydroxylase } \\
\text { deficiency, external genital virilization of } \\
\text { Prader } 3 \text { corresponding phenotypically to } \\
\text { "AIS3" }\end{array}$ \\
\hline L-3 & 3 & $\begin{array}{l}\text { labia majora labioscrotal } \\
\text { swellings }\end{array}$ & normal female & $40 ; 4$ & normal female $(46, X X)$ \\
\hline ARD709 & 1 & testis gonad & ambiguous (AIS3) & $6 ; 5$ & Met775Ile \\
\hline ARD29 I & 1 & testis gonad & $\begin{array}{l}\text { normal female } \\
\text { (CAIS) }\end{array}$ & $18 ; 4$ & Phe794Ser, negative androgen binding \\
\hline ARD53 I & 1 & testis gonad & $\begin{array}{l}\text { normal female } \\
\text { (CAIS) }\end{array}$ & 35,2 & Ala765Thr, negative androgen binding \\
\hline ARD557 & 1 & testis gonad & $\begin{array}{l}\text { normal female } \\
\text { (CAIS) }\end{array}$ & $6 ; 6$ & $\begin{array}{l}\text { donor splice site exon } 2 / \text { intron } 2 \text {, negative } \\
\text { androgen binding }\end{array}$ \\
\hline ARD842 & 1 & testis gonad & $\begin{array}{l}\text { normal female } \\
\text { (CAIS) }\end{array}$ & $38 ; 0$ & $\begin{array}{l}26 \text { bp deletion exon I ( }|4|-\mid 50) \text {, } \\
\text { frameshift, premature stop codon, negative } \\
\text { androgen binding }\end{array}$ \\
\hline ARDI004 & 1 & testis gonad & $\begin{array}{l}\text { normal female } \\
\text { (CAIS) }\end{array}$ & $17 ; 4$ & Val866Met, negative androgen binding \\
\hline N-ST4 & 1 & abdominal skin & normal male & $46 ; 0$ & no functional data \\
\hline N-LS I 2 & 1 & forearm skin & normal male & $36 ; 0$ & no functional data \\
\hline
\end{tabular}

Fibroblast strains; microarray dataset in whichh they have been analysed (I - 3); ARD or GS, patient strain-ID; F, normal male foreskin fibroblasts; S, normal male scrotal fibroblasts. L-I-3 represent the only samples with a 46, XX karyotype. N-ST4 and N-LSI 2 are of extragenital origin. ARD5I5 was derived from a $46, X Y$ male with ambiguous genitalia due to a $5 \alpha$ reductase type II defect rather than AIS. Normal ranges for androgen (methyltrienolone) binding: $B_{\max }$ (binding capacity): $13-116 \mathrm{fmol} / \mathrm{mg}$ protein; $\mathrm{K}_{\mathrm{d}}$ (dissociation constant): 0.03-0.13 $\mathrm{nM}$.

system suggested by Sinnecker [10] wherein phenotypically male genitalia are scored AIS 1 , while female external genitalia are scored AIS 5 (Fig. 2B). Stringent filtering conditions of the combined data sets reduced the number of transcripts from 612 to 259; however, altering the stringency of the filtering conditions and the number of transcripts used did not significantly change the clustering pattern of the individual samples (data not shown).

Clustering separated the 72 experiments into two major subgroups. The righthand major branch included predominantly patients with female external genitalia while most of the patients in the lefthand major branch had normal male or highly virilized external genitalia (Fig. 2A and 2B). All but one of the samples with CAIS (AIS 5) and a normal 46, XX female clustered in the right ("female") branch (Fig. 2A). Interestingly, the one exception expressed a wild type AR in a portion of cells due to somatic mosaicism (ARD465). Two skin fibroblast samples from normal males derived from regions without an obvious androgen induced sexual dimorphism (abdomen, forearm) also clustered in the right ("female") branch. The left ("male") major branch contained all genital skin fibroblasts derived from normal male controls and 8 of 10 microarry experiments reflecting patients with higher degrees of virilization due to partial AIS (AIS 2). This cluster also included a fibroblast sample from an individual with $5 \alpha$-reductase type II deficiency, a defect which results in ambiguous genitalia due to lack of conversion of testosterone to dihydrotestosterone. This individual presumably possesses a wild-type AR, meaning that androgen signaling pathways remained intact. Three of the four labioscrotal fibroblast samples from individuals with AIS 3 phenotypes with significant genital ambiguity clustered in the "female" major branch and the remaining in the "male" one (Fig. 2). Of note, clustering did not 
appear to be influenced by array type or RNA referencetype, indicating that normalization procedures did not influence data quality.

Structure within the cluster dendrogram suggested that there was some residual influence of topology on gene expression in the samples. In the righthand "female" branch, fibroblast samples derived from AIS gonads clustered separately from all skin-derived samples (Fig. 2A). Similarly, the lefthand "male" branch showed a subcluster that contained all the foreskin-derived fibroblasts, including the AIS 2 fibroblasts originating from the foreskin. Interestingly, this branch also contained two strains of labia minora fibroblasts from two 46, XX individuals, one of whom had ambiguous genitalia (Prader stage 3 ) due to 21-hydroxylase deficiency (female pseudohermaphroditism), while the other individual was a normal female. Since the labia minora are analogous to the urethral folds that participate in penile morphogenesis, this finding suggests that topographic origin influenced expression within the selected set of genes of these two samples more than AR signaling. In some cases, the anatomic origin of biopsy was not well documented (table 1). This might explain why some samples did not cluster as expected (e.g., ARD380 and ARD659, Fig. 2A) although other factors might have contributed to these findings.

We also wanted to reconsider whether the new topologyindependent, AR gene list better classified samples than our previous gene list that did not control for the locations from which samples were harvested. After removing the microarray experiments that were used to define either the previous [8] or the new gene set by SAM, the remaining samples were clustered. As expected, in case of both gene lists the samples sorted into two main branches that separated primarily male and female samples (Fig. 3A and 3B). Moreover, when we considered only topology-controlled samples originating from the labioscrotal swellings excluding the mosaic samples and those of insufficiently described biopsy localization, the mean AISgrades between the two branches differed significantly using both gene lists (new gene list: AIS-grades: $2.5 \pm 0.55$ (male); $4.17 \pm 1.12$ (female); $\mathrm{p}<0.001$ by t-test; previous gene list (Holterhus et al. 2003): AIS-grades: $1.6+0.79$ (male); $3.3+1.6$ (female); $\mathrm{p}<0.01$ by t-test). However, in contrast to the new gene set, the previous gene set that did not account for topology misclassified many individuals. It resulted in incorrectly female classification of most of the highly virilized individuals with AIS 2 (3 of 4 individuals $=75 \%)$ and of a large fraction of the normal male scrotal fibroblast controls ( 3 of 7 individuals $=43 \%$ ) (Fig. $3 \mathrm{~A}$ and $3 \mathrm{~B})$. The new gene set misclassified only one individual with AIS 2 (ARD306).

\section{Biological processes in the AR-dependent gene expression program}

We performed a systematic analysis for enrichment of genes belonging to defined biological processes and cellular pathways using the PANTHER classification system [11]. PANTHER classifies genes by their functions, based on published experimental evidence and on evolutionary relationships. The 612 significant transcripts corresponded to 527 named transcripts, of which PANTHER recognized 440 unique gene IDs. Several related biological processes were significantly over-represented in the AR-dependent gene list including "control of cell proliferation and differentiation" ( $\mathrm{p}=0.00001$, "developmental processes" ( $\mathrm{p}=0.00013)$ and "cell cycle control" ( $\mathrm{p}=$ 0.00041) (table 2). Analysis of cellular pathways also revealed several interesting signaling pathways including "angiogenesis" ( $\mathrm{p}=0.00001)$ and WNT-signaling" ( $\mathrm{p}=$ 0.00002 ) (table 3 ). These processes and pathways were reflected in the major branches of the cluster dendrogram revealing differential expression in the phenotypically male and female samples (Figures 2C and 2D). For instance, samples in the "male" branch showed high expression of CCN1 (cyclin 1), CCND1 (cyclin D1), IGF2 (insulin-like growth factor 2), IGFBP5 (Insulin-like growth factor binding protein 5), MYC (V-myc myelocytomatosis viral oncogene homolog), MAFF (V-maff musculoaponeurotic fibrosarcoma oncogene homolog $\mathrm{F}$ ), EGFR (epidermal growth factor receptor), PTPN3 (protein tyrosine phophatase), MET (hepatocyte growth factor receptor) and several other genes important in cell growth and proliferation (Fig. 1 and 2A). Transcripts expressed at high levels in the "female" branch included ANAPC7 (anaphase promoting complex subunit 7), FZD8 (frizzled homolog 8) and FZD6 (frizzled homolog 6) (Fig. 1).

The AR-dependent gene set showed enrichment for a number of genes related to maintenance and modification of tissue shape and structural identity (Tables 2 and 3 , and Fig. 2D). Genes that were up-regulated in the predominately male branch were SDC1 (syndecan 1), FMOD (fibromodulin) and ADAMTS2 (A disintegrin-like and metalloproteinase with thrombospondin type 1 motif, 2). Those up-regulated mainly in the female branch included ADAM12 (A disintegrin and metalloproteinase 12), TNC (tenascin C), CSPG2 (chondroitin sulfate proteoglycan 2, versican), FBN1 (fibrillin 1), ELN (elastin), COL5A2 (collagen, type $\mathrm{V}$, alpha2), ECM2 (extracellular matrix protein 2, female organ and adipocyte specific), DAG1 (dystroglycan 1), and SPOCK (sparc/osteonectin, cwcV kazal-like domains proteoglycan, testican).

\section{Confirmation of selected transcripts by RT-PCR}

Four transcripts were selected for confirmation of the microarray data by semi-quantitative RT-PCR: TNC (tenascin), FZD8 (frizzled 8), ADAM12 (ADAM metal- 

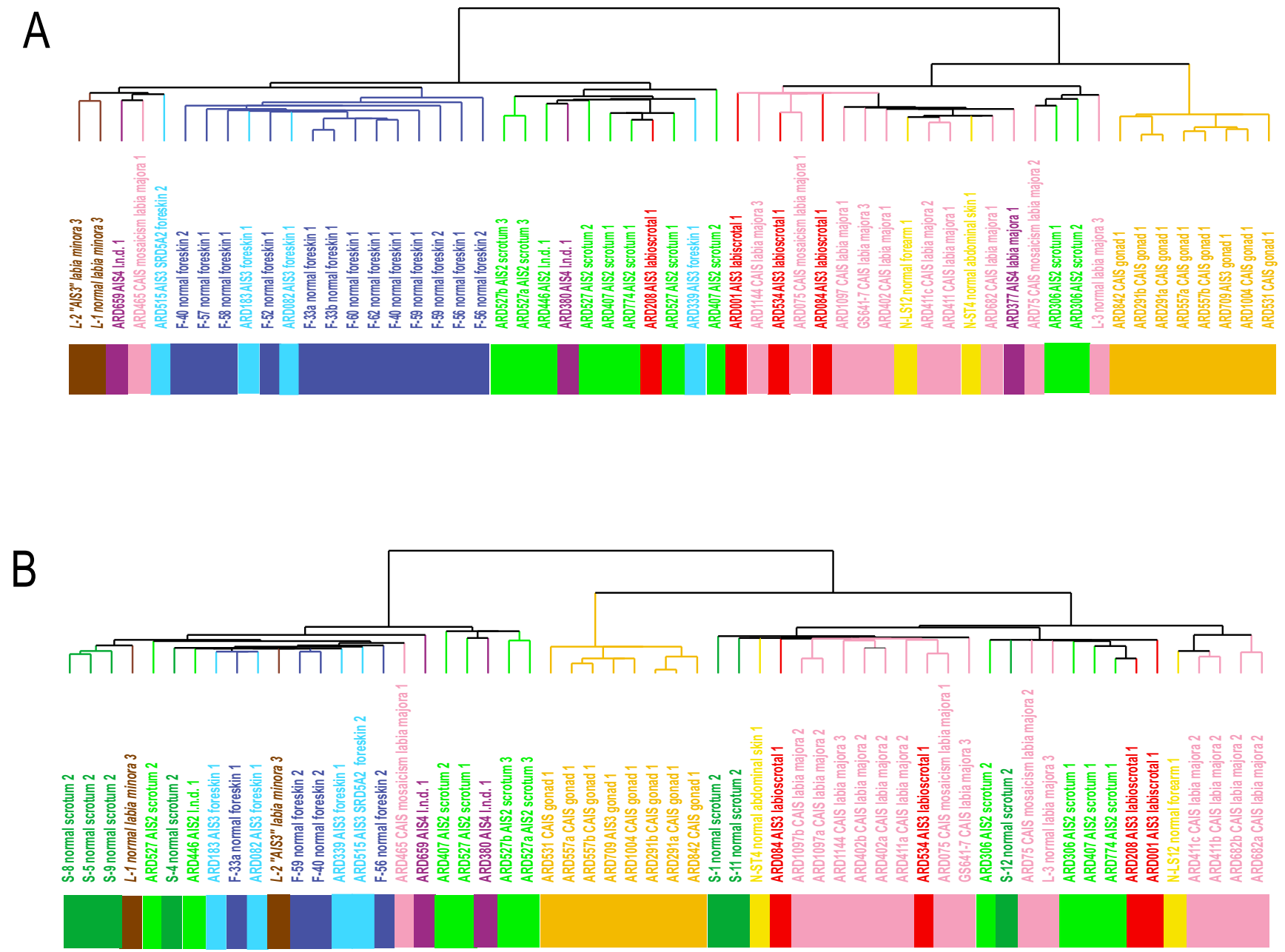

Figure 3

Experiment clustering with topography controlled (A) - versus previous (B) [8] gene set. Hierarchical clustering analyses of microarray experiments using the new topology-independent AR gene list (A) and our previous gene list [8] that did not control for topology of biopsy location (B). In each of the two clusters $A$ and $B$, the microarray experiments used to generate the underlying list of significant genes by SAM were removed before clustering resulting 57 remaining experiments in (A) and 58 in (B), respectively. The color code is the same as in Fig. 2. Based on the previous gene set, several highly virilized AIS 2 patients and normal scrotal fibroblasts were incorrectly classified female.

lopeptidase 12), and CSPG2 (chondroitin sulfate proteoglycane 2, versican). RNA from the labia majora fibroblasts derived from CAIS affected individuals (ARD402, ARD411, ARD682, ARD1097), as well as four samples of the normal male scrotal fibroblasts (S4, S5, S8, S9) were used for analysis. In agreement with the microarray data, TNC, FZD8, ADAM12 and CSPG2 show significantly higher expression in the labia majora fibroblasts compared to the scrotal fibroblasts (Fig. 4).

\section{Discussion}

We demonstrate reproducible, lasting differences in gene expression in cultured fibroblasts harvested from analogous structures in the genital tissues of normal males and
46, XY CAIS females. This unique model system, in which samples were matched for genotype and topology, allows identification of gene expression programs predominantly influenced by AR signaling, likely during genital morphogenesis. By selecting fibroblasts derived from the labioscrotal swellings in normal and CAIS individuals, we were able to largely exclude androgen-independent mechanisms as a cause of systematic differences of baseline gene expression. While we had identified a related set of genes previously using fibroblast cells that had not been matched for site of origin, the significant differences between our previous and current gene sets implies that topology significantly influenced gene expression in our original set. This finding is instructive for future studies 
Table 2: Biological processes

\begin{tabular}{|c|c|c|c|}
\hline Biological process & $\begin{array}{l}\text { Detected number } \\
\text { of genes in SAM list }\end{array}$ & $\begin{array}{l}\text { Expected number of genes } \\
\text { based on NCBI reference list }\end{array}$ & P-value \\
\hline Cell proliferation and differentiation & 40 & 18.46 & 0.00001 \\
\hline Intracellular signaling cascade & 35 & 15.59 & 0.00001 \\
\hline Protein phosphorylation & 28 & 12.07 & 0.00005 \\
\hline Protein modification & 40 & 20.81 & 0.00008 \\
\hline Developmental processes & 61 & 37.57 & 0.00013 \\
\hline Biological process unclassified & 157 & 193.59 & 0.00024 \\
\hline Cell cycle control & 18 & 7.16 & 0.00041 \\
\hline Cell structure and motility & 36 & 20.29 & 0.00076 \\
\hline Signal transduction & 86 & 61.52 & 0.0008 \\
\hline Extracellular matrix protein-mediated signaling & 6 & 1.09 & 0.00091 \\
\hline
\end{tabular}

AR-dependent biological processes identified by PANTHER (Thomas et al. 2003) [I I] as significantly over-represented in the SAM-list compared to the NCBI human reference gene list (table limited to genes with $p<0.001$ )

seeking to identify the effects of inactivating mutations of single genes in tissues or cells cultured from tissues. Gene expression profiles can be influenced by obvious genotypic differences, such as the presence of an $\mathrm{X}$ or Y chromosome [6] but also by subtle differences including differences in topology [9].

The transcripts we have identified appear to be highly relevant to male and female external genital morphogenesis. When used to classify an independent set of fibroblasts cultured from normal male, normal female, and PAISaffected individuals, this gene set performed considerably better than our previous set in which we had not controlled for differences in topology. The observation that fibroblasts derived from the labia majora of normal female external genitalia clustered with AIS derived samples from the same site demonstrates the primacy of developmental androgen actions in influencing gene expression in this set of genes, and possibly in external genital morphogenesis. Furthermore, our findings suggest that gene expression profiling of genital fibroblasts might be correlated with phenotype in vivo and could serve as a diagnostic marker of the extent of developmental androgenization, although many more cases will need to be analyzed, and other analytic methods will need to be used to identify an ideal classifier.

Chang and co-workers have demonstrated that fibroblasts cultured from different regions of the body retain position-specific expression signatures [9]. Our work confirms the presence of lasting, topology-influenced differences in gene expression in human fibroblasts. Controlling for the site from which genital fibroblasts were derived significantly altered the set of genes we identified as differing between normal and CAIS-derived fibroblasts. Despite that control, signatures of topology remained in our new

Table 3: Cellular pathways

\begin{tabular}{lcc}
\hline Cellular pathway & $\begin{array}{c}\text { Detected number of } \\
\text { genes in SAM-list }\end{array}$ & $\begin{array}{c}\text { Expected number of genes } \\
\text { based on NCBI reference list }\end{array}$ \\
\hline Angiogenesis & 16 & 4.12 \\
Wnt signaling & 20 & 6.71 \\
T cell activation & 10 & 2.26 \\
Inflammation mediated by chemokine and cytokine signaling & 16 & 0.00001 \\
pathway & & 0.00002 \\
Endothelin signaling pathway & 8 & 0.00012 \\
Muscarinic acetylcholine receptor I and 3 signaling pathway & 5 & 0.00038 \\
Integrin signaling pathway & 12 & 0.00145 \\
Alpha adrenergic receptor signaling pathway & 4 & 0.00145 \\
EGF receptor signaling pathway & 8 & 0.00202 \\
Unclassified & 370 & 0.00209 \\
Axon guidance mediated by semaphorins & 5 & 0.44 \\
PDGF signaling pathway & 9.53 \\
Ras pathway & 9 & 2.56 \\
\hline
\end{tabular}

AR-dependent cellular pathways identified by PANTHER (Thomas et al. 2003) [II] as significantly over-represented in the SAM-list compared to the NCBI human reference gene list (table limited to genes with $p<0.0$ ) 


\section{Tenascin C}

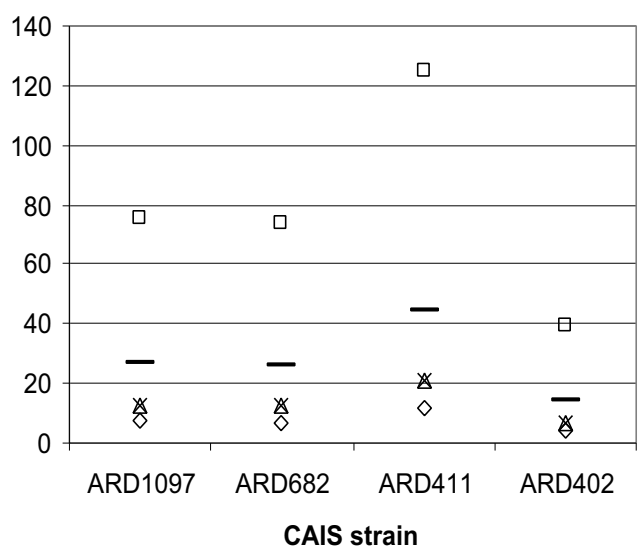

Frizzled homolog 8 (Drosophila)

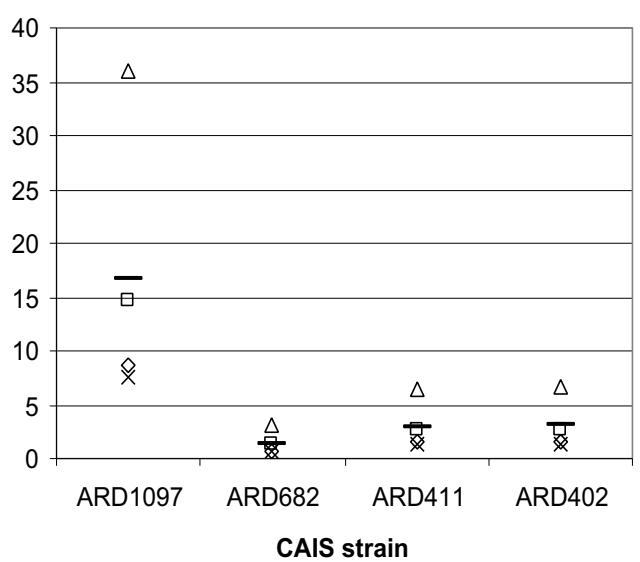

\section{Chondroitin sulfate proteoglycane 2 (versican)}

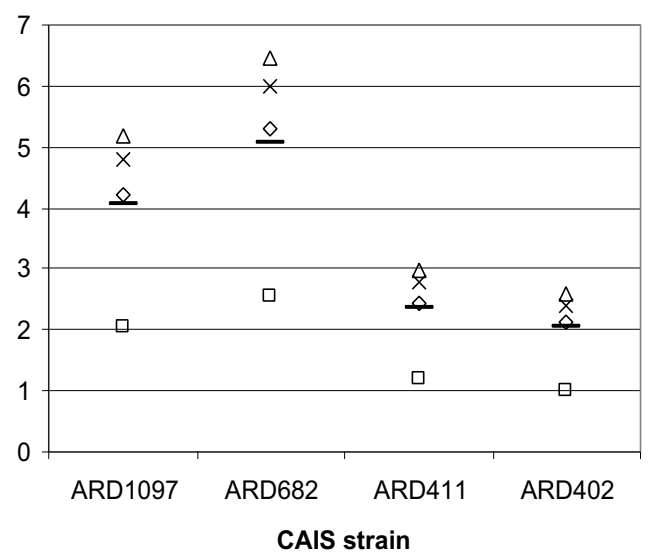

ADAM metallopeptidase domain 12

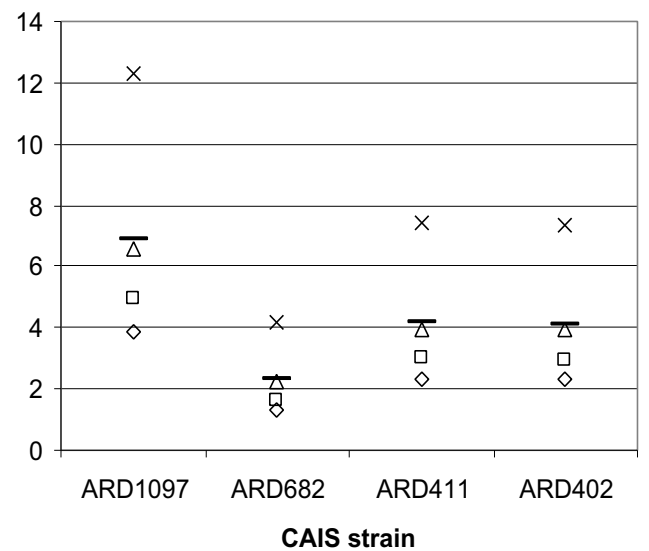

\section{scrotum 4}

\section{scrotum 5}

\section{$\triangle$ scrotum 9}

\section{$\times$ scrotum 8}

- mean

\section{Figure 4}

Verification of selected genes by RT-PCR. The ratio of transcript levels of TNC (Tenascin), FZD8 (Frizzled 8), ADAMI2 (ADAM metallopeptidase 12), and CSPG2 (Chondroitin sulfate proteoglycane 2, versican) comparing CAIS cell lines and normal scrotal cell lines. Semi-quantitative RT-PCR was performed on four samples of normal male scrotal fibroblasts (S4, S5, S8, S9), as well as on four samples of labia majora derived from CAIS patients (ARD402, ARD4II, ARD682, ARDI097). Differences in expression levels between different cell lines were calculated according to the $\Delta \Delta$-CT method [19]. The $y$-axis represents the ratios of expression levels of CAIS labia majora fibroblasts divided by those from scrotal fibroblasts. 
gene set. For instance, normal male fibroblasts and CAISderived gonadal fibroblasts each clustered as distinct entities in the "male" and "female" arms, respectively. Thus, even in our stringently selected fibroblast samples and gene set, topology remains evident in the gene expression profile. Although Chang and co-workers have found that HOX gene signatures account for much of the topological gene classifier, our gene list lacked HOX genes, implying that traces of topology remain encoded in other sets of transcripts. Together with the results of Chang et al., our work demonstrates substantial heterogeneity in gene expression in cultured fibroblasts that can be influenced by topology and, in the case of our unique fibroblast samples, AR signaling. We suspect that other signaling pathways, possibly including other steroid hormones or growth factors, can program lasting changes gene expression in fibroblasts. Whether these differences in gene expression can influence development of associated tissues in vivo remains to be elucidated. Regardless, our data suggests one possible model system for teasing out the effects of mutations in single genes on global gene expression patterns in cells cultured from in vivo models.

The biological processes and the cellular pathways enriched in the AR-dependent gene set could provide some insights into the role of AR-mediated programming of fibroblasts in male and female external genital differentiation. For instance, activation of several growth signaling pathways in male derived fibroblasts and WNT signaling pathways in female genital fibroblasts, e.g., FZD8 (Fig. 1, 2 and 4), might signal their important roles in genital development. The same could be true for genes contributing to maintenance and modification of tissue shape and structural identity, e.g., versican, tenascin, and ADAM12 (Fig. 1, 2 and 4). It is unclear, however, whether expression of these genes in cultured fibroblasts reflects their expression in vivo, whether expression is necessary for maintenance of external genital structure, or whether these pathways are reactivated as a result of cell culture. Moreover, it cannot be excluded that potentially higher cumulative estrogens during development and postnatal life in the AIS patients until the time of genital skin biopsy may have had additional influences on gene expression. It is possible that the gene expression programs have little to do with genital development; however, the finding that many genes related to cell and tissue structure and integrity is tantalizing. We identified many genes of the cytoskeletal network (KRT5, 19, 25A, MYO10) and of the surrounding extracellular matrix (TNC, SPOCK, CSPG2, COL5A2) in the AR-dependent gene expression data set [12-14]. Additional in vivo work including strategies comprising early embryonal urogenital tissues will clarify whether genes identified using our approach are important in external genital morphogenesis.

\section{Conclusion}

Our data demonstrate the existence of large-scale ARdependent gene expression programs in fibroblasts cultured from human external genitalia. These programs are influenced neither by differences in sex chromosomes nor by the topographic differences between the genital tubercle and the labioscrotal swellings. Improved classification of individuals with partial and complete androgen insensitivity syndrome with this gene list compared to a set we had identified previously suggests that androgen programming must have played a key role in establishing these programs. Therefore, the detected genes represent valuable targets for unraveling human external genital differentiation and the role of androgen therein.

\section{Methods}

The study was approved by the ethical committee of the University of Lübeck, Germany. Informed consent was obtained from the control subjects, affected individuals or their parents.

\section{Cell strains}

A total of 51 different primary fibroblast cultures were analyzed (table 1). Genital fibroblasts were established from scrotal skin biopsies from normal males undergoing orchidopexy or testis biopsy (scrotal fibroblasts) or from circumcisions (foreskin fibroblasts). Normal female genital skin fibroblasts were established from individuals undergoing genital plastic surgery (labia majora fibroblasts). Genital skin fibroblasts of patients with disorders of sex development (DSD, [15]), mostly AIS, were obtained either for diagnostic reasons or during reconstructive surgery (foreskin-derived or labioscrotal-derived or labial fibroblasts) or following gonadectomy (gonadal fibroblasts). Extra-genital skin fibroblasts were obtained from phenotypically normal male individuals. For gene expression profiling, fibroblasts of the 3rd to 7th passage were cultured to confluency (wherein they entered $\mathrm{G}_{0}$ arrest), as described previously [8].

\section{RNA-isolation and cDNA-labeling}

Protocols for mRNA and total RNA preparation, and cDNA labeling are available at [16]. Either $2 \mu \mathrm{g}$ of mRNA (dataset 1) or $50 \mu \mathrm{g}$ of total RNA (datasets 2 and 3) from genital fibroblasts were reverse transcribed and labeled with Cy5. Labeled cDNAs from genital fibroblasts were mixed with Cy-3-labeled reference RNA from Stategene (datasets 2 and 3 ) or a pooled reference of RNA from fibroblasts and 11 immortalized cell lines (dataset 1, see [8]).

\section{Microarrays and hybridizations}

Poly L-lysine coated (data set $1=19$ new hybridized micoarrays plus 22 previously published microarrays [8] and dataset $2=24$ new hybridized microarrays) or Corn- 
ing glass slide (data set $3=7$ new hybridized microarrays) spotted cDNA-microarrays containing more than 44,000 elements corresponding to approximately 26,000 unique genes (UniGene clusters) were used for gene expression analysis (for details, see [16]). Hybridizations were performed using equal amounts of Cy3 and Cy5 labeled cDNAs according to previously published protocols for 14-18 hours over night at $65^{\circ} \mathrm{C}$. Hybridized microarrays were scanned using a GenePix4000 array scanner and analyzed with GenePix Pro software (Axon Instruments, Inc., Union City, CA).

\section{Microarray data analysis}

To identify genes differentially expressed in homologous tissues of normal male and CAIS female derived fibroblasts, we restricted our analysis to samples derived from the labioscrotal swellings (scrotum or labia majora), that were hybridized on poly-lysine coated slides against Strategene reference RNA (data set 2). Only spots with fluorescence signals of 1.5-fold over array background in either the experimental or reference channel were considered. The Cy5/Cy3 fluorescence ratios for all genes in each array were normalized to obtain an average absolute $\log _{2}$ red/green ratio of 0 and the fluorecence ratios for each gene were mean centered. The Significance Analysis of Microarrays (SAM) procedure [17] was used to identify transcripts with statistically significant differences in expression levels between normal and CAIS samples. Identified transcripts were used to perform hierarchical clustering analysis of 72 microarray experiments performed on fibroblasts derived from normal males, and individuals affected with PAIS and CAIS. To correct for differences in the reference RNA (dataset 1 versus dataset 2 and 3 ) and slide coating (dataset 1 and 2 versus dataset 3), normalized $\log _{2}$ red/green ratios for single genes were centered by the mean in each of the three datasets separately according to a previously published procedure [18]. Centered data from the three data sets were combined and again were mean-centered for each gene. In the combined dataset, only genes with measurable data in $85 \%$ of the 72 experiments were considered. Restriction of the analysis to genes which had absolute values of normalized $\log _{2}$ red/green ratios $\geq 1.0$ in at least 5 experiments of the sample set of 72 microarrays resulted in 259 transcripts. Microarray experiments and genes were organized by hierarchical clustering, using the Pearson correlation metric and average linkage clustering [19]. TreeView software was used to visualize the results [19]. Supplementary information on each of the microarray experiments and all datasets are available online $[22,23]$. For figure 1 please see additional files $1,2,3,4$, for figure 2 , please see additional files 5, 6, 7, 8 .

\section{Functional classification of sexually dimorphic genes}

SAM identified transcripts were loaded into the PANTHER (Protein ANalysis THrough Evolutionary Relationships) database. Enrichment of biological processes and cellular pathways in the AR dependent gene list was determined by comparing the number of genes observed in the SAMlist for each process or pathway to the number of genes expected based on the NCBI Homo sapiens reference gene list. PANTHER determines a p-value based on binominal statistics, describing the probability that the number of detected genes occurred randomly, with a p-value $<0.05$ indicating significance [11]. Since many pathways can be identified, we focused our discussion on a subset in biological processes with $\mathrm{p}<0.001$ and in cellular pathways with $\mathrm{p}<0.01$.

\section{RT-PCR analyses}

Semi-quantitative RT-PCR was performed using FAM (F) labeled probes with dabcyl (DB) quencher. RT-PCR was performed on four samples of normal male scrotal fibroblasts (S4, S5, S8, S9) as well as on four samples of labia majora derived from CAIS patients (ARD402, ARD411, ARD682, ARD1097). Exon-spanning primers and probes (TIB Molbiol, Berlin, Germany) had the following sequences: Tenascin: TNC-forward: ACAgTgggACAgCAggTgACT, Tm: $59.6^{\circ} \mathrm{C}$; TNC-reverse: CAggTTgACACggTgACAgTTC, $\mathrm{Tm}: 59.4^{\circ} \mathrm{C}$, TNC labeled probe: $\mathrm{F}$ TACCACAATggCAgATCCTTCTCCACCT-DB, Tm: $66.7^{\circ} \mathrm{C}$; Frizzled 8: FZD8-forward: gTgCAgCgAAgggACACTTg, Tm: 61.0 ${ }^{\circ} \mathrm{C}$; FZD8-reverse: TCCTCAgCCAACAgAAATTAACg, $\mathrm{Tm}: 58.7^{\circ} \mathrm{C}$, FZD8 labeled probe: F-AggTTCCCACCCCTTCACAgTgTTgA-DB, Tm: $67.9^{\circ} \mathrm{C}$; ADAM metallopeptidase 12: ADAM12-forward: CCTggCACCCCTCAgACC, Tm: $\quad 60.9^{\circ} \mathrm{C}, \quad$ ADAM12-reverse: gTACAAAAAACTCCAACTggAgCTg, Tm: $59.4^{\circ} \mathrm{C}$, ADAM12 labeled probe: $\mathrm{F}$ TCCACACCAAgTgCCCAgATCCAC-DB, Tm: $67.0^{\circ} \mathrm{C}$; Chondroitin sulfate proteoglycane 2, versican: CSPG2forward: TgTCTCACgAAgAACAAATgTTTgT, Tm: $58.0^{\circ} \mathrm{C}$, CSPG2-reverse: gggTCTCCAATTCTCgTATTgCA, Tm: $59.8^{\circ} \mathrm{C}$, CSPG2 labeled probe: F-TgCCATCAgTCCAACggAAgTCATgCT-DB, Tm: $69.3^{\circ} \mathrm{C}$; TATA-binding protein (TBP) was used to normalize expression values. TBP primers were TBP-forward: CACgAACCACggCACTg-ATT, TBPreverse: TTTTCTTgCTgCCAgTCTggAC; TBP-labeled probe: F-TgTgCAC-AggAgCCAAgAgTgAAgA-DB. Reverse transcription was performed using the Ambion RETROscript kit according to the manufacturer's recommendations with minor modifications. $2 \mu \mathrm{g}$ of total RNA, $0.5 \mu \mathrm{M}$ dNTPs, $5 \mu \mathrm{M}$ random decamers and 200 units of MMLVRT polymerase were used per reaction. Heat denaturation was performed for $3 \mathrm{~min}$ at $85^{\circ} \mathrm{C}$ preceding reverse transcription for $1 \mathrm{hr}$ at $44^{\circ} \mathrm{C}$ and subsequent inactivation for $10 \mathrm{~min}$ at $92^{\circ} \mathrm{C}$. C-DNA corresponding to $12 \mathrm{ng}$ of initial total RNA served as template for amplification reactions. $0.4 \mu \mathrm{M}$ of each primer, $0.17 \mu \mathrm{M}$ of specific probe and Taq- 
Man Universal PCR mastermix (Applied Biosystems, Foster City, CA, USA) including the Taq polymerase were added to a final volume of $25 \mu$ l. Initial denaturation was performed at $95^{\circ} \mathrm{C}$ for $10 \mathrm{~min}$ followed by $1 \mathrm{~min}$ cycling intervals at $60^{\circ} \mathrm{C}$ using a RotorGene RG-3000 cycler (Corbett-Research, Sydney, Australia). Differential transcription levels between all different cell lines were calculated according to the $\Delta \Delta$-CT method [20].

\section{Abbreviations}

$\mathrm{AR}$, androgen receptor; AIS, androgen insensitivity syndrome; CAIS, complete androgen insensitivity syndrome; PAIS, partial androgen insensitivity syndrome

\section{Authors' contributions}

All authors read and approved the final manuscript. PMH was responsible for the experimental design, assessment of clinical information on AIS-patients (including clinical examination), culturing fibroblasts, RNA-works and microarray hybridizations (data set 1), functional characterization of AR in fibroblast samples, data analysis, and he was the leading writer of the manuscript. UD planned and performed culturing fibroblasts, RNA-works, microarray hybridizations, microarray data analysis and management (data set 2 and 3), and contributed essentially to writing the manuscript. RW helped essentially with the microarray strategies and data analysis and contributed to writing the manuscript. He was also responsible for RTPCR. ARU was responsible for clinical assessment of patients and contributed to writing the manuscript. In particular, she contributed the 46 , XX fibroblasts. JHB performed microarray data analysis and wrote the manuscript. LW was responsible for clinical assessment of several patients, and he obtained many fibroblast biopsies, especially the normal scrotal controls, and contributed to writing the manuscript. SK assessed many patients clinically and contributed to writing the manuscript. HUS was reponsible for clinical assessment of several patients (data set 3), contributed functional characterization of AR in fibroblast samples (several of the androgen binding studies) and contributed to writing the manuscript. JD was indispensable for microarray data analysis, central data management at Stanford University and the web repository of data. He also helped in creating the figures for the web supplement. FR assessed many patients clinically, took part in data analysis and was one of the writers of the manuscript. $\mathrm{OH}$ was an important senior clinical supervisor of genital phenotype assessment, he contributed most of the molecular data of the androgen receptor gene to the study and he was a key person in data interpretation with respect to androgen insensitivity syndrome. JDB was the senior writer of the manuscript. He supervised the experimental strategy, including all statistical issues with respect to the microarray data.

\section{Additional material}

\section{Additional file 1}

Text file to visualize data for figure 1 together with additional files 2, 3 and 4 in TreeView [19].

Click here for file

[http://www.biomedcentral.com/content/supplementary/1471-

2164-8-376-S1.txt]

\section{Additional file 2}

Cluster file to visualize data for figure 1 together with additional files 1 , 3 and 4 in TreeView [19].

Click here for file

[http://www.biomedcentral.com/content/supplementary/1471-

2164-8-376-S2.cdt]

\section{Additional file 3}

Array tree file to visualize data for figure 1 together with additional files 1, 2 and 4 in TreeView [19].

Click here for file

[http://www.biomedcentral.com/content/supplementary/1471-

2164-8-376-S3.atr]

\section{Additional file 4}

Gene tree file to visualize data for figure 1 together with additional files 1, 2 and 3 in TreeView [19].

Click here for file

[http://www.biomedcentral.com/content/supplementary/1471-

2164-8-376-S4.gtr]

\section{Additional file 5}

Text file to visualize data for figure 2 together with additional files 6,7 and 8 in TreeView [19].

Click here for file

[http://www.biomedcentral.com/content/supplementary/1471-

2164-8-376-S5.txt]

\section{Additional file 6}

Cluster file to visualize data for figure 2 together with additional files 5 , 7 and 8 in TreeView [19].

Click here for file

[http://www.biomedcentral.com/content/supplementary/1471-

2164-8-376-S6.cdt]

\section{Additional file 7}

Array tree file to visualize data for figure 2 together with additional files 5, 6 and 8 in TreeView [19].

Click here for file

[http://www.biomedcentral.com/content/supplementary/14712164-8-376-S7.atr]

\section{Additional file 8}

Gene tree file to visualize data for figure 2 together with additional files 5, 6 and 7 in TreeView [19].

Click here for file

[http://www.biomedcentral.com/content/supplementary/14712164-8-376-S8.gtr] 


\section{Acknowledgements}

The study was supported by grants from the Deutsche Forschungsgemeinschaft (DFG) (KFO I I I/I-C, Ho2073/5-I and Ho2073/5-2 to P.M.H.), and NIH grant CAI I I782 (J.D.B.). We thank Genevieve Vidanes, Christine Marschke, and Dagmar Struve for excellent technical assistance. We are grateful to the scientists and staff of the Stanford Microarray Facility and the Stanford Microarray Database without whom the project would not have been realized. We also thank Gunnar Cario, MD, for helpful discussion on normalization strategies.

Paul-Martin Holterhus, Uta Deppe, Ralf Werner, Jan-Hendrik Bebermeier, and Olaf Hiort are members of the DFG (Deutsche Forschungsgemeinschaft)-funded Clinical Research Group KFOI I I: "Intersex - From Gene to Gender"

Paul-Martin Holterhus, Ralf Werner, Annette Richter-Unruh, Lutz Wünsch, Susanne Krege, Felix Riepe, Olaf Hiort are members of the German BMBF (Bundesministerium für Bildung und Forschung) network for Rare Diseases "DSD - Disorders of Sex Development"

\section{References}

I. Wilson JD, Griffin JE, Leshin M, George FW: Role of gonadal hor mones in development of the sexual phenotypes. Hum Genet I98I, 58:78-84.

2. Quigley CA, De Bellis A, Marschke KB, el-Awady MK, Wilson EM, French FS: Androgen receptor defects: historical, clinical, and molecular perspectives. Endocr Rev 1995, 16:27I-321.

3. Hiort $O$, Holterhus PM: The molecular basis of male sexual differentiation. Eur J Endocrinol 2000, 142:101-10.

4. MacLaughlin DT, Donahoe PK: Sex determination and differentiation. N Engl J Med 2004, 350:367-78.

5. Grumbach MM, Conte FA, Hughes IA: Disorders of Sex Differentiation. In Williams Textbook of Endocrinology IOth edition. Edited by: Larsen PR, Kronenberg HM, Melmed SM, Polonsky MS. Philadelphia, Saunders; 2002:771-1002.

6. Whitney AR, Diehn M, Popper SJ, Alizadeh AA, Boldrick JC, Relman $D A$, Brown PO: Individuality and variation in gene expression patterns in human blood. Proc Natl Acad Sci USA 2003, I00:1896-1901.

7. Yang $X$, Schadt EE, Wang S, Wang H, Arnold AP, Ingram-Drake L, Drake TA, Lusis AJ: Tissue-specific expression and regulation of sexually dimorphic genes in mice. Genome Res 2006, 16:995-1004.

8. Holterhus PM, Hiort O, Demeter J, Brown PO, Brooks JD: Differential gene-expression patterns in genital fibroblasts of normal males and 46, XY females with androgen insensitivity syndrome: evidence for early programming involving the androgen receptor. Genome Biol 2003, 4:R37.

9. Chang HY, Chi JT, Dudoit S, Bondre C, van de Rijn M, Botstein D, Brown PO: Diversity, topographic differentiation, and positional memory in human fibroblasts. Proc Natl Acad Sci USA 2002, 99: I 2877-12882.

10. Sinnecker GH, Hiort O, Nitsche EM, Holterhus PM, Kruse K: Functional assessment and clinical classification of androgen sensitivity in patients with mutations of the androgen receptor gene. Eur J Pediatr 1997, I 56:7-I4.

II. Thomas PD, Kejariwal A, Campbell MJ, Mi H, Diemer K, Guo N, Ladunga I, Ulitsky-Lazareva B, Muruganujan A, Rabkin S, Vandergriff JA, Doremieux O: PANTHER: a browsable database of gene products organized by biological function, using curated protein family and subfamily classification. Nucleic Acids Res 2003, 3 I:334-34 I [http://www.pantherdb.org/].

12. Kresse H, Schonherr E: Proteoglycans of the extracellular matrix and growth control. I Cell Physiol 200I, I 89:266-274.

13. Chiquet-Ehrismann R: Tenascins. Int J Biochem Cell Biol 2004, 36:986-990.

14. Chanut-Delalande H, Bonod-Bidaud C, Cogne S, Malbouyres M, Ramirez F, Fichard A, Ruggiero F: Development of a functional skin matrix requires deposition of collagen $\mathbf{V}$ heterotrimers. Mol Cell Biol 2004, 24:6049-6057.
15. Hughes IA, Houk C, Ahmed SF, Lee PA, LWPES Consensus Group; ESPE Consensus Group: Consensus statement on management of intersex disorders. Arch Dis Child 2006, 91 :554-563.

16. The Brown lab [http://cmgm.stanford.edu/pbrown/]

17. Tusher VG, Tibshirani R, Chu G: Significance analysis of microarrays applied to the ionizing radiation response. Proc Natl Acad Sci USA 200I, 98:5I I6-5I 21 .

18. Bullinger L, Dohner K, Bair E, Frohling S, Schlenk RF, Tibshirani R, Dohner $\mathrm{H}$, Pollack JR: Use of gene-expression profiling to identify prognostic subclasses in adult acute myeloid leukemia. $\mathrm{N}$ Engl J Med 2004, 350:1605-1616.

19. Eisen MB, Spellman PT, Brown PO, Botstein D: Cluster analysis and display of genome-wide expression patterns. Proc Nat Acad Sci USA 1998, 95:|4863-14868.

20. Livak KJ, Schmittgen TD: Analysis of relative gene expression data using real-time quantitative PCR and the 2(-delta delta C(T)) method. Methods 25:402-408.

2I. Elmlinger MW, Mayer I, Schnabel D, Schuett BS, Diesing D, Romalo G, Wollmann HA, Weidemann W, Spindler KD, Ranke MB, Schweikert HU: Decreased expression of IGF-II and its binding protein, IGF-binding protein-2, in genital skin fibroblasts of patients with complete androgen insensitivity syndrome compared with normally virilized males. I Clin Endocrinol Metab 200I, 86:474I-4746.

22. Supplemental information [http://microarray-pubs.stanford.edu/ AIS-2/]

23. Stanford Genomic Resources [http://genome-www.stan ford.edu/]

24. SOURCE [http://smd-www.stanford.edu/cgi-bin/source/source Search]
Publish with Bio Med Central and every scientist can read your work free of charge

"BioMed Central will be the most significant development for disseminating the results of biomedical research in our lifetime. "

Sir Paul Nurse, Cancer Research UK

Your research papers will be:

- available free of charge to the entire biomedical community

- peer reviewed and published immediately upon acceptance

- cited in PubMed and archived on PubMed Central

- yours - you keep the copyright

Submit your manuscript here:

http://www.biomedcentral.com/info/publishing_adv.asp
BiolMedcentral 